\title{
Autophagy in hepatic diseases
}

\section{Editorial}

Autophagy or cellular "self-eating" is a normal physiological process dealing with cellular destruction. Autophagy is highly conserved evolutionary homeostatic mechanism. Autophagy is multifaceted process that completely concerned with the normal functioning by protein degradation, turnover of intracellular components as well as the management of stress responses. Autophagy initiates by induction of a small vesicular sac called the isolation membrane or phagophore that elongates and subsequently encloses a portion of cytoplasm resulting in to the formation of a double membrane structure called autophagosome. Mitochondria participates in autophagosome biogenesis through its outer membrane which fuses with a lysosome leading to the degradation of the enclosed materials. Autophagy is intracellular bulk degradation systems that succeed membrane trafficking mechanism for delivering cellular component and organelles for degradation with in lysosome/vacuole. ${ }^{2}$ For the degradation of cellular protein, there are only two pathways one is proteasome and second is lysosome even if protein aggregates themselves consequently that protein aggregation directly damaged the purpose of the ubiquitin-proteasome system.., Autophagy acts as an essential programmed cellular process in elimination of pathogen and cytosolic aggregated proteins and subcellular organelles. The process leads to transportation of aggregated protein to lysosomes for degradation. Autophagy is significantly involved in the degradation of intracellular bacteria, tumor suppression, antigen presentation, and cell survival and death. ${ }^{4}$ Autophagy induction and execution involves a series of biochemical events through activation or regulation of several genes like Atg, uncoordinated 51-like kinase 1 (ULK1) complex, nucleation with the Beclin-1-class III phosphatidylinositol 3-kinase (PI3K) complex, and microtubule-associated protein light chain 3 (LC3).

Studies dated back in 1960s showed identification of lysosomes and the discovery of autophagy in liver. It was defined to be the process of degradation of intracellular proteins and organelles in lysosomes. Studies in liver also demonstrated that nutritional status of hepatocytes regulates autophagy and that circulating hormones modulate the catabolic autophagic activity in the liver. Intensive hepatic autophagy studies in years demonstrated the importance of lysosome-mediated degradation in maintaining liver homeostasis in normal physiological conditions. Studies also revealed the adequate response of liver to stress inducers like proteotoxicity, metabolic dysregulation, infection and carcinogenesis. Autophagic malfunctions have been found associated with common liver pathogenic diseases indicating that chemical manipulation of autophagy might be potentially therapeutic in nature. ${ }^{7}$ Recent researches show the involvement of autophagy in liver diseases. In certain liver diseases the level of autophagy is varied to the specific function. In liver ischemia reperfusion and liver surgery, the level of autophagy is increased and acts a prosurvival function. Infections of hepatitis $\mathrm{B}$ and $\mathrm{C}$ are also characterized by increased autophagy. During acute liver injury also autophagy is increased and functions in cell survival or death dependent manner. In alcoholic and fatty liver disease, autophagy is decreased marked by degradation of protein aggregates and of damaged organelles as well as lipid degradation. Autophagy is decreased also in hepatocellular carcinoma and has an anti-tumor therapeutic approach. ${ }^{7,8}$ Thus autophagy has intriguing roles in liver disease, tumorigenesis, and
Volume 4 Issue I - 2016

\author{
Siddhartha Kumar Mishra \\ Department of Zoology, Dr. Harisingh Gour Central University, \\ India
}

Correspondence: Siddhartha Kumar Mishra, Department of Zoology, School of Biological Sciences, Dr. Harisingh Gour Central University, Sagar- 470003 (MP) India, Tel +91-7582265004,Email siddharthakm@yahoo.com

Received: December 28, 2015 | Published: January 27, 2016

anti-tumor therapeutics against hepatocellular carcinoma. Chronic hepatitis B infection induced autophagosomes in liver cells harboring low (Tg08) and high (Tg05) replication levels of the HBV DNA. ${ }^{9}$ HBx also plays a crucial role in this HBV-induced autophagy which was also confirmed from Huh7.5 cells (HBx negative) not showing autophagy. ${ }^{9}$ An interesting in vitro study in HepG2 cells indicated that ginsenoside Rk1 (inhibitor of G(1) phase) have anti-tumor activity and that it inhibited telomerase and induce cell death. ${ }^{10}$ The study recognized that Rk1-induced autophagy might also include conversion of LC3-I to LC3-II and monodansylcadaverine (MDC) and also that autophagy inhibitors in combination with $\mathrm{Rk} 1$ can be used as an effective anti-cancer treatments of liver. ${ }^{10}$ Yet the exact molecular mechanism how HBV and $\mathrm{HBx}$ regulates autophagy is not constant and not well understood.

Autophagy responses in acute liver injury are more unusual as in the two classic models of acute liver injury such as the concanavalin $\mathrm{A}$ and the lipopolysaccharide/D- galactosamine induced acute hepatitis. ${ }^{8,11}$ Autophagy was enhanced in both models as demonstrated in mice liver involving the specific mechanism of pregnane $\mathrm{X}$ receptor (PXR). Autophagy has also been implicated in hepatocyte lipid metabolism through a process called macrolipophagy. ${ }^{12}$ The hepatocyte-specific at g7-knockout mice had markedly increased hepatic lipid and decreased hepatic autophagy indications. ${ }^{12,13}$ Hepatic cancer has been genetically linked to autophagy malfunction with autophagy regulation in tumorigenesis. Hepatocellular carcinoma (HCC) has clearly demonstrated that autophagy is a tumor suppressor mechanism. Disruption of beclin-1 has a high frequency of spontaneous HCC and the expression of several autophagic genes like ATG5 and ATG7, and B-cell leukemia/lymphoma (Bcl)-xL has been intriguing in $\mathrm{HCC} .{ }^{14,15}$

Autophagic and non-autophagy pathways can be considered as competitive determinants of cell fate. However, if the homeostatic balance between autophagy and apoptosis is lost, either pathway can potentially drive cellular demise. Some epigenetic modifiers are also involved in the regulation of autophagy and potentiate the efficacy of traditional therapeutics. Therapeutic approaches aiming at modulation of autophagy have been tested and have shown interesting results with promising therapeutic management. Autophagy modulation has confirmed specificity and beneficial impacts in liver diseases. Thus, understanding the novel functions of autophagy control may allow developing potential therapeutic approaches for liver cancer management. 


\section{Acknowledgments}

None.

\section{Conflicts of interest}

The authors have no conflict of interest for this research.

\section{Funding}

None.

\section{References}

1. Hailey DW, Rambold AS, Satpute-Krishnan P, et al. Mitochondria supply membranes for autophagosome biogenesis during starvation. Cell. 2010;141(4):656-667.

2. Dunn WA Jr. Autophagy and related mechanisms of lysosome-mediated protein degradation. Trends Cell Biol. 1994;4(4):139-143.

3. Bence NF, Sampat RM, Kopito RR. Impairment of the ubiquitin-proteasome system by protein aggregation. Science. 2001;292(5521):1552-1555.

4. Baumeister W, Walz J, Zuhl F, et al. The proteasome: paradigm of a self-compartmentalizing protease. Cell. 1998;92(3):367-380.

5. Levine B, Kroemer G. Autophagy in the pathogenesis of disease. Cell. 2008;132(1):27-42.

6. Mehrpour M, Esclatine A, Beau I, et al. Autophagy in health and disease. 1. Regulation and significance of autophagy: an overview. $\mathrm{Am}$ J Physiol Cell Physiol. 2010;298(4):C776-785.
7. Schneider JL, Cuervo AM. Liver autophagy: much more than just taking out the trash. Nat Rev Gastro Hepatol. 2014;11(3):187-200.

8. Rautou PE, Mansouri A, Lebrec D, et al. Autophagy in liver diseases. $J$ Hepatol. 2010;53(6):1123-1134.

9. Sir D, Tian Y, Chen WL, Ann DK, et al. The early autophagic pathway is activated by hepatitis B virus and required for viral DNA replication. Proc Nat Acad Sci USA. 2010;107(9):4383-4388.

10. Ko H, Kim YJ, Park JS, et al. Autophagy inhibition enhances apoptosis induced by ginsenoside Rk1 in hepatocellular carcinoma cells. Biosci Biotechnol Biochem. 2009;73(10):2183-2189.

11. Chang CP, Yang MC, Liu HS, et al. Concanavalin A induces autophagy in hepatoma cells and has a therapeutic effect in a murine in situ hepatoma model. Hepatology. 2007;45(2):286-296.

12. Singh R, Kaushik S, Wang Y, et al. Autophagy regulates lipid metabolism. Nature. 2009;458(7242):1131-1135.

13. Czaja MJ. Autophagy in health and disease. 2. Regulation of lipid metabolism and storage by autophagy: pathophysiological implications. Am J Physiol Cell Physiol. 2010;298(5):C973-C978.

14. Qu X, Yu J, Bhagat G, et al. Promotion of tumorigenesis by heterozygous disruption of the beclin 1 autophagy gene. J Clin Invest. 2003;112(12):1809-1820.

15. Ding ZB, Shi YH, Zhou J, et al. Association of autophagy defect with a malignant phenotype and poor prognosis of hepatocellular carcinoma. Can Res. 2008;68(22):9167-9175. 